\title{
BINARY SUBTREES WITH FEW LABELED PATHS
}

\author{
RODNEY G. DOWNEY, NOAM GREENBERG, CARL G. JOCKUSCH, JR.,
} AND KEVIN G. MILANS

\begin{abstract}
We prove several quantitative Ramseyan results involving ternary complete trees with $\{0,1\}$-labeled edges where we attempt to find a complete binary subtree with as few labels as possible along its paths. One of these is used to answer a question of Simpson's in computability theory; we show that there is a bounded $\Pi_{1}^{0}$ class of positive measure which is not strongly (Medvedev) reducible to $\mathrm{DNR}_{3}$; in fact, the class of 1-random reals is not strongly reducible to $\mathrm{DNR}_{3}$.
\end{abstract}

\section{INTRODUCTION}

There have been many fruitful interactions between combinatorics and computability theory. Examples include new combinatorial proofs of classical results such as Mileti's proof of the canonical Ramsey theorem [12], Montalbán's newly devised invariants for infinite linear orderings [13], Kierstead's algorithmic online version of Dilworth's Theorem [9], and Füredi et. al. on inverting the difference operator [7. This paper is another example of such an interaction.

We study edge-labelings of rooted trees. A tree is ternary if each non-leaf has 3 children and binary if each non-leaf has 2 children. A tree is complete if all leaves are at the same distance from the root, and the depth of a complete tree is the distance between the root and a leaf. If $T$ is a complete ternary tree of depth $n$, we define $\mathcal{B}(T)$ to be the set of all binary subtrees of $T$ that are complete with depth $n$. A tree $T$ is edge-labeled if each edge in $T$ is assigned a label from the set $\{0,1\}$. We define $\mathcal{T}_{n}$ to be the set of all ternary, complete, edge-labeled trees of depth $n$.

Rod Downey acknowledges support from the Marsden Fund and a James Cook Fellowship. Carl Jockusch thanks the Marsden Fund for partial financial support for his travel to Wellington when the research for this paper started. Noam Greenberg also acknowledges support from the Marsden Fund. 
If $T \in \mathcal{T}_{n}, r$ is the root of $T$, and $\sigma$ is a leaf in $T$, then reading the elements along the path from $r$ to $\sigma$ in $T$ gives a path-label $x \in\{0,1\}^{n}$, and we say that $\sigma$ has path-label $x$. We define $L(T)$ to be the set of all path-labels in $T$. Given $T \in \mathcal{T}_{n}$, we wish to find a subtree $S \in \mathcal{B}(T)$ that minimizes $|L(S)|$. For each $T \in \mathcal{T}_{n}$, let $f(T)=\min \{|L(S)|: S \in \mathcal{B}(T)\}$, and for each $n$, let $f(n)=\max \left\{f(T): T \in \mathcal{T}_{n}\right\}$.

The combinatorial thrust of our paper is to study the behavior of $f(n)$ as $n$ grows. In Section 2 , we show that $\lim _{n \rightarrow \infty}(f(n))^{1 / n}$ exists; our bounds on $f(n)$ imply that this limit has a value between $2^{1 / \log _{2} 3} \approx 1.548$ and 2 . In Section 3 we show that if $c<\sqrt{\log _{2}(4 / 3)} \approx 0.644$, then there is a constant $\gamma$ such that $f(n) \leq \gamma 2^{n-c \sqrt{n}}$. Consequently, the ratio $f(n) / 2^{n}$ tends to zero as $n$ grows. This result has the following Ramsey interpretation: for large $n$, every edge-labeled complete ternary tree of depth $n$ admits a complete binary subtree of depth $n$ whose path-labels constitute an arbitrarily small fraction of the space of all possible path-labels. In Section 4 we prove that $f(n) \geq 2^{(n-3) / \log _{2} 3}$. Our techniques lead to a solution of a problem in computability theory and effective randomness.

In his survey paper [19] on mass problems and randomness, Simpson asked whether for all $k \geq 3$, the Medvedev degree of $\mathrm{DNR}_{k}$ bounds the Medvedev degree of every $\Pi_{1}^{0}$ class of positive measure. We give precise definitions in Section 5. but the gist of the question the question concerns comparing the computational difficulty of diagonalization with a constant bound with that of constructing a set which is effectively random. The full background and motivation for this question, which we answer in the negative in this paper, can be found in Section 5 . In fact, we obtain the stronger result that the class of Kurtz random reals is not Medevedev reducible to $\mathrm{DNR}_{3}$.

The solution of the problem involves continuous maps from the ternary version $3^{\mathbb{N}}$ of Cantor space to the standard binary one $2^{\mathbb{N}}$. It turns out that once the computability aspects of the problem are untangled, the key to our solution is purely combinatorial. Sections 2,3 and 4 present the combinatorial results and can 
be read independently of Section 5 , which presents the application in computability theory.

\section{Some facts and a Question about $f$}

We begin by collecting a few simple facts about $f(n)$. The following recursive bounds on $f(n)$ are instructive.

\section{Proposition 2.1.}

(1) If $n$ is a positive integer, then $f(n+1) \leq 2 f(n)$.

(2) If $r$ and $s$ are positive integers, then $f(r+s) \geq f(r) f(s)$.

Proof. To prove (1), let $T \in \mathcal{T}_{n+1}$ be a tree with root $r$, and let $T_{0}$ and $T_{1}$ be subtrees of $T$ rooted at two children of $r$. Since $T_{0}, T_{1} \in \mathcal{T}_{n}$, by induction each $T_{i}$ has a binary subtree $S_{i} \in \mathcal{B}\left(T_{i}\right)$ containing at most $f(n)$ path-labels, and combining these subtrees with the root of $T$ yields a binary subtree of $T$ with at most $2 f(n)$ path-labels.

To prove (2), let $R \in \mathcal{T}_{r}$ be a tree in which each $R^{\prime} \in \mathcal{B}(R)$ contains at least $f(r)$ path-labels, and let $S \in \mathcal{T}_{s}$ be a tree in which each $S^{\prime} \in \mathcal{B}(S)$ contains at least $f(s)$ path-labels. Obtain $T \in \mathcal{T}_{r+s}$ by attaching a copy of $S$ at each leaf in $R$. Each binary subtree of $T$ contains at least $f(r) f(s)$ labels.

Proposition 2.1 is helpful in computing the first few values of $f$.

\section{Proposition 2.2.}

(1) If $1 \leq i \leq 4$, then $f(i)=i$.

(2) $6 \leq f(5) \leq 8$.

Proof. We leave part (1) as an exercise for the reader. For part (2), note that $f(2) f(3) \leq f(5) \leq 2 f(4)$ by Proposition 2.1 and apply part (1).

Proposition 2.1 has further consequences. First, the upper bound $f(n+1) \leq$ $2 f(n)$ shows that $f(n) / 2^{n}$ is a non-increasing sequence, and because $f(n) \geq 0$, it 
4 englishROD DOWNEY, NOAM GREENBERG, CARL JOCKUSCH, AND KEVIN G. MILANS

follows that $\lim _{n \rightarrow \infty} f(n) / 2^{n}$ exists. Indeed, we shall see that this limit is zero. Another consequence of Proposition 2.1 is that $\lim _{n \rightarrow \infty}(f(n))^{1 / n}$ exists.

Proposition 2.3. If $a_{n}=(f(n))^{1 / n}$ and $\beta=\sup \left\{a_{n}\right\}$, then $\lim a_{n}=\beta$.

Proof. Note that $\beta \leq 2$ since $f(n) \leq 2^{n}$. Fix $\varepsilon>0$ and choose $m$ so that $a_{m} \geq$ $\beta-\varepsilon / 2$. Let $n$ be large, and divide $n$ by $m$ to get a quotient $q$ and remainder $r$. Iteratively applying Proposition 2.1, we have that

$$
f(n) \geq(f(m))^{q} f(r)=\left(a_{m}\right)^{m q} f(r) \geq\left(a_{m}\right)^{n-r}
$$

Hence, we have that $a_{n} \geq\left(a_{m}\right)^{1-r / n}$. Because $\left(a_{m}\right)^{1-r / n} \rightarrow a_{m}$ as $n \rightarrow \infty$, it follows that there exists $n_{0}$ such that $n \geq n_{0}$ implies that $a_{n} \geq a_{m}-\varepsilon / 2$. Therefore, for each $n \geq n_{0}$, we have that $\beta-\varepsilon \leq a_{n} \leq \beta$.

We thank colleagues in a seminar for pointing out that this result can also be proved by noting that $g(n)=\log f(n)$ is a superadditive function, i.e. $g(m+n) \geq$ $g(m)+g(n)$ for all positive integers $m, n$. Also $g(n) / n$ is bounded. It then follows from a result known as Fekete's Lemma (see [16], \#98, page 23, solution on page 198) that $\lim _{n} g(n) / n$ exists and equals $\sup g(n) / n$. Restating this in terms of $f$ completes the proof.

It follows from the previous two propositions that

$$
\lim _{n \rightarrow \infty}(f(n))^{1 / n} \geq(f(3))^{1 / 3}=\sqrt[3]{3} \geq 1.442
$$

We shall see in Corollary 4.4 that

$$
\lim _{n \rightarrow \infty}(f(n))^{1 / n} \geq 2^{\frac{1}{\log _{2} 3}} \geq 1.548
$$

On the other hand, the best known upper bound is the trivial bound

$$
\lim _{n \rightarrow \infty}(f(n))^{1 / n} \leq 2
$$

This leads to the main open problem regarding bounds on $f(n)$. 
Problem 2.4. What is $\lim _{n \rightarrow \infty}(f(n))^{1 / n}$ ?

\section{AN UPPER BOUND ON $f(n)$}

We begin collecting results needed to establish our upper bound on $f(n)$. The following proposition is central to the task at hand. It is implicit in the proof of Theorem 6 of [8], which is a sort of forerunner of our Theorem 5.2. It was also stated explicitly by Robert Goldblatt in [5] (bottom of page 561) where it was applied to solve a problem in modal logic. It may well have occurred elsewhere in the literature, but we are not aware of work previous to our paper on bounding the number of path-labels in binary subtrees of edge-labeled ternary trees.

Proposition 3.1. Let $T$ be a complete ternary tree of depth $n$. If each leaf in $T$ is colored red or blue, then there exists $S \in \mathcal{B}(T)$ such that all leaves in $S$ share a common color.

Proof. Let $r$ be the root of $T$ and let $T_{1}, T_{2}$, and $T_{3}$ be the subtrees of $T$ rooted at the children of $r$. By induction, each tree $T_{j}$ contains a subtree $S_{j} \in \mathcal{B}\left(T_{j}\right)$ in which all leaves share a common color. By the pigeonhole principle, at least two of the $S_{j}$ contain leaves of the same color. Combining these with the root of $T$, we obtain $S \in \mathcal{B}(T)$ as required.

Proposition 3.1 has a very useful consequence. When $X \subseteq\{0,1\}^{n}$, we let $\bar{X}$ be the complementary set $\{0,1\}^{n} \backslash X$.

Corollary 3.2. If $T \in \mathcal{T}_{n}$ and $X$ is a subset of $\{0,1\}^{n}$, then there exists $S \in \mathcal{B}(T)$ such that either $L(S) \subseteq X$ or $L(S) \subseteq \bar{X}$.

Proof. Label a leaf $\sigma$ in $T$ red if the path-label from the root of $T$ to $\sigma$ is in $X$ and blue otherwise. By Proposition 3.1, there exists $S \in \mathcal{B}(T)$ such that all leaves share a common color. If this color is red, then $L(S) \subseteq X$. Otherwise, $L(S) \subseteq \bar{X}$.

Our strategy to bound $f(n)$ from above is as follows. We prove our bound by induction, and to prove $f(n)$ is small, we use that $f(m)$ is small for a carefully chosen number $m<n$. 
Consider $T \in \mathcal{T}_{n}$. We first find a complete binary subtree $S^{\prime}$ of depth $m$ such that $\left|L\left(S^{\prime}\right)\right| \leq f(m)$. For each leaf $\sigma$ in $S^{\prime}$, let $T_{\sigma}$ be the subtree of $T$ rooted at $\sigma$. Note that each $T_{\sigma}$ is a member of $\mathcal{T}_{n-m}$. To extend $S^{\prime}$ to a complete binary subtree of depth $n$, we wish to find a family of complete binary subtrees $S_{\sigma} \in \mathcal{B}\left(T_{\sigma}\right)$ such that $\left|\bigcup_{\sigma} L\left(S_{\sigma}\right)\right|$ is as small as possible.

So the key for this process is arguing not only that given a single edge-labeled ternary tree we can find a complete binary subtree with few path-labels, but actually that given a family of edge-labeled ternary trees, we can find corresponding binary subtrees such that the total number of path-labels used in all of the binary subtrees together is small. Corollary 3.2 gives some control over the path-labels that appear in the binary subtrees. In order to find the binary subtrees, we apply Corollary 3.2 numerous times with different subsets of $\{0,1\}^{n}$. We are particularly interested in applying Corollary 3.2 to families of subsets of $\{0,1\}^{n}$ with a certain structure.

Definition 3.3. Let $\alpha \in[0,1]$, and let $\Upsilon$ be a ground set. Two partitions $\{X, \bar{X}\}$ and $\{Y, \bar{Y}\}$ of $\Upsilon$ are $\alpha$-orthogonal if all four of the cross intersections: $X \cap Y$, $X \cap \bar{Y}, \bar{X} \cap Y$, and $\bar{X} \cap \bar{Y}$, have size at least $\alpha|\Upsilon| / 4$. A family of partitions $\mathcal{X}$ is $\alpha$-orthogonal if each pair of distinct partitions in $\mathcal{X}$ is $\alpha$-orthogonal.

While we construct large $\alpha$-orthogonal families for an arbitrary ground set $\Upsilon$, we apply our construction in the case $\Upsilon=\{0,1\}^{n}$. Note that if $\alpha<1$ and $X$ and $Y$ are chosen independently and uniformly at random from all subsets of a large ground set, then $\{X, \bar{X}\}$ and $\{Y, \bar{Y}\}$ are $\alpha$-orthogonal with high probability. This suggests a natural way of constructing large $\alpha$-orthogonal families. We shall need Chernoff's inequality.

Theorem (Chernoff's Inequality). (See [14, Theorem 4.2].) Let $Z_{1}, Z_{2}, \ldots, Z_{t}$ be mutually independent random indicator variables where $Z_{l}=1$ with probability $p_{l}$ and $Z_{l}=0$ with probability $1-p_{l}$, let $Z=\sum_{l=1}^{t} Z_{l}$, and let $\mu=\mathbf{E}[Z]=\sum_{l=1}^{t} p_{l}$. If $0 \leq \delta \leq 1$, then $\operatorname{Pr}[Z<(1-\delta) \mu]<e^{-\mu \delta^{2} / 2}$. 
Lemma 3.4. Let $\alpha \in(0,1)$, and $\Upsilon$ be a ground set of size $t$. There exists a family of pairwise $\alpha$-orthogonal partitions of $\Upsilon$ of size at least

$$
\left\lfloor\frac{\sqrt{2}}{2}\left(e^{\frac{(1-\alpha)^{2}}{16}}\right)^{t}\right\rfloor .
$$

Proof. Let $r=\left\lfloor\frac{\sqrt{2}}{2} e^{\frac{(1-\alpha)^{2}}{16} t}\right\rfloor$. For each $1 \leq j \leq r$, choose a subset $X_{j} \subseteq \Upsilon$ uniformly and independently at random. We claim that with positive probability, $\left\{X_{i}, \overline{X_{i}}\right\}$ and $\left\{X_{j}, \overline{X_{j}}\right\}$ are $\alpha$-orthogonal when $i \neq j$. In particular, this implies that with positive probability, the partitions are all distinct and that $\left\{\left\{X_{j}, \overline{X_{j}}\right\}: 1 \leq j \leq r\right\}$ is an $\alpha$-orthogonal family of size $r$, which implies that some such family exists. Let $\mathcal{X}=\left\{\left\{X_{j}, \overline{X_{j}}\right\}: 1 \leq j \leq r\right\}$.

For each pair $\{i, j\}$ with $1 \leq i<j \leq r$, let $A_{i j}$ be the event that one of the four cross intersections between $\left\{X_{i}, \overline{X_{i}}\right\}$ and $\left\{X_{j}, \overline{X_{j}}\right\}$ has size less than $\alpha \frac{t}{4}$, and let $A=\bigcup_{i j} A_{i j}$, so that $A$ is the event that $\mathcal{X}$ is not an $\alpha$-orthogonal family. We show that $\operatorname{Pr}[A]<1$.

Of course $\operatorname{Pr}[A] \leq \sum_{i j} \operatorname{Pr}\left[A_{i j}\right]$. Similarly, we have that $\operatorname{Pr}\left[A_{i j}\right] \leq 4 p$, where $p$ is the probability that $|X \cap Y|<\alpha \frac{t}{4}$ where $X \subseteq \Upsilon$ and $Y \subseteq \Upsilon$ are chosen uniformly and independently at random. For each $x \in \Upsilon$, let $Z_{x}$ be the random indicator variable for the event that $x \in X \cap Y$, and let $Z=\sum_{x} Z_{x}$, so that $Z=|X \cap Y|$. Note that the $Z_{x}$ are mutually independent random indicator variables and $Z_{x}=1$ with probability $1 / 4$. By Chernoff's inequality,

$$
p=\operatorname{Pr}\left[Z<\alpha \frac{t}{4}\right]<e^{-(t / 4)(1-\alpha)^{2} / 2}=e^{-\frac{(1-\alpha)^{2}}{8} t} .
$$

It follows that

$$
\operatorname{Pr}[A] \leq \sum_{i j} \operatorname{Pr}\left[A_{i j}\right] \leq \sum_{i j} 4 p=4\left(\begin{array}{l}
r \\
2
\end{array}\right) p<2 r^{2} e^{-\frac{(1-\alpha)^{2}}{8} t} \leq 1
$$

and hence $\operatorname{Pr}[A]<1$ as required. 
It is possible to construct larger $\alpha$-orthogonal families using more sophisticated probabilistic tools, such as the Lovász Local Lemma. However, these larger families do not give substantial improvements to our bounds on $f(n)$, so we omit them.

Lemma 3.5. Let $\varepsilon>0$ and let $k=\log _{2}\left(\varepsilon^{-2} \ln 4\right)$. If $T_{1}, \ldots, T_{r} \in \mathcal{T}_{n}$ and $n \geq$ $\log _{2}(r)+k$, then there are binary subtrees $S_{j} \in \mathcal{B}\left(T_{j}\right)$ such that

$$
\left|\bigcup_{j} L\left(S_{j}\right)\right| \leq\left(\frac{3}{4}+\varepsilon\right) 2^{n} .
$$

Proof. Let $\alpha=1-4 \varepsilon$, so $\alpha<1$. By Lemma 3.4 there is a family $\mathcal{X}$ of pairwise $\alpha$-orthogonal partitions of $\{0,1\}^{n}$ of size

$$
\begin{aligned}
\left\lfloor 2^{-1 / 2} e^{\varepsilon^{2} 2^{n}}\right\rfloor & \geq\left\lfloor 2^{-1 / 2} e^{\varepsilon^{2}\left(r \varepsilon^{-2} \ln 4\right)}\right\rfloor \\
& =\left\lfloor 2^{-1 / 2} e^{r \ln 4}\right\rfloor \\
& =\left\lfloor 2^{2 r-1 / 2}\right\rfloor \\
& >2^{r}
\end{aligned}
$$

Fix an arbitrary linear ordering on $\{0,1\}^{n}$. For each $\{X, \bar{X}\} \in \mathcal{X}$, we apply Corollary 3.2 to each of the trees $T_{1}, \ldots, T_{r}$. Let $D_{\{X, \bar{X}\}}$ be the subset of $\left\{T_{1}, \ldots, T_{r}\right\}$ consisting of those trees $T$ for which Corollary 3.2 produces a binary subtree $S \in \mathcal{B}(T)$ where $L(S) \subseteq \min \{X, \bar{X}\}$, where the minimization is with respect to the chosen ordering on $\{0,1\}^{n}$.

Because $|\mathcal{X}|>2^{r}$, there exist distinct partitions $\{X, \bar{X}\}$ and $\{Y, \bar{Y}\}$ in $\mathcal{X}$ with $D_{\{X, \bar{X}\}}=D_{\{Y, \bar{Y}\}}$. Let $D=D_{\{X, \bar{X}\}}$. For each $T_{j}$, we select $S_{j} \in \mathcal{B}\left(T_{j}\right)$ as follows. If $T_{j} \in D$, then we may choose $S_{j} \in \mathcal{B}\left(T_{j}\right)$ such that $L\left(S_{j}\right) \subseteq \min \{X, \bar{X}\}$. Alternately, if $T_{j} \notin D$, then we may choose $S_{j} \in \mathcal{B}\left(T_{j}\right)$ such that $L\left(S_{j}\right) \subseteq \max \{Y, \bar{Y}\}$. 
Note that none of the $S_{j}$ contains a path-label in $Z=\max \{X, \bar{X}\} \cap \min \{Y, \bar{Y}\}$. Moreover, because $\mathcal{X}$ is $\alpha$-orthogonal, we have that $|Z| \geq(\alpha / 4) 2^{n}$. It follows that

$$
\left|\bigcup_{j} L\left(S_{j}\right)\right| \leq 2^{n}-(\alpha / 4) 2^{n} \leq\left(\frac{3}{4}+\varepsilon\right) 2^{n} .
$$

We remark that the hypothesis $n \geq \log _{2}(r)+k$ cannot be relaxed beyond reducing $k$. Indeed, suppose that $r=2^{n}$ and index the ternary trees by vectors in $\{0,1\}^{n}$. If each $T_{x}$ is edge-labeled so that $L\left(T_{x}\right)=\{x\}$, then $L(S)=\{x\}$ for each $S \in \mathcal{B}\left(T_{x}\right)$. Consequently, regardless of which subtrees are chosen, $\bigcup_{x} L\left(S_{x}\right)=\{0,1\}^{n}$.

Our main result (Theorem 3.7 below) asserts that for sufficiently small constants $c>0$ the function $f$ is $O\left(2^{n-c \sqrt{n}}\right)$. We now briefly outline the proof of this result. Using induction, assume the result for some $m<n$ (which we will now need to pick carefully, given $n$ ), and given $T$, pick a complete binary subtree $S^{\prime}$ of depth $m$ such that $\left|L\left(S^{\prime}\right)\right|$ is bounded by $\gamma 2^{m-c \sqrt{m}}$ for an appropriate choice of the constant $\gamma$. Now we have two kinds of path-labels in $S^{\prime}$ : those that occur often (in the proof, at least $2^{c \sqrt{m}}$ many times), and those that do not. If a path-label $x$ appears often, it doesn't matter how we choose to extend $S^{\prime}$ at leaves $\sigma$ with path-label $x$, because the total number of path-labels for all leaves of $S$ extending any such $\sigma$ will be limited. And if a label $x$ does not appear often, then we can apply Lemma 3.5 to obtain trees $S_{\sigma}$ extending all the leaves of $S^{\prime}$ which are labeled by $x$, with a bounded total number of labels.

Our next lemma is technical and determines how we choose the depth $m$ of subtree on which we apply induction. Because we apply Lemma 3.5 to a collection of $2^{m}$ trees of depth $n-m$, we need $n-m$ to be large. On the other hand, we will replace $\sqrt{m}$ with $\sqrt{n}$ in some of our bounds, so we want $\sqrt{m}$ to be close to $\sqrt{n}$.

Lemma 3.6. Let $c>0$ and $k>0$. If $n$ is a sufficiently large integer, then there exists an integer $m$ with $1 \leq m<n$ such that $n-m \geq c \sqrt{m}+k$ and $\sqrt{n}-\sqrt{m} \leq c$. 
10 englishROD DOWNEY, NOAM GREENBERG, CARL JOCKUSCH, AND KEVIN G. MILANS

Proof. For positive $x \in \mathbb{R}$, let $y(x)=x-c \sqrt{x}-(k+1)$. We have $y(x) \rightarrow \infty$, so for large enough real $x$, we can let $h(x)=\sqrt{x}-\sqrt{y(x)}$. High-school algebra shows that $h(x)=\frac{n(x)}{d(x)}$, where $n(x)=(c+(k+1) / \sqrt{x})$ and $d(x)=$ $(1+\sqrt{1-c / \sqrt{x}+(k+1) / x})$, for all sufficiently large $x$. Hence $h(x) \rightarrow c / 2$ as $x$ grows. Therefore $h(x) \leq c$ when $x$ is sufficiently large. Let $n$ be large enough so that $h(n) \leq c$ and $y(n)>0$, and let $m=\lceil y(n)\rceil$. Note that $m<n$ since $y(n)<n-1$. Because $m-1<y(n) \leq m$, we have that

$$
n-m=n-(m-1)-1 \geq n-y(n)-1=c \sqrt{n}+k \geq c \sqrt{m}+k .
$$

Similarly, we have $\sqrt{n}-\sqrt{m} \leq \sqrt{n}-\sqrt{y(n)}=h(n) \leq c$. Finally, note that because $y(n)>0$, we have that $1 \leq m$.

Theorem 3.7. If $0 \leq c<\sqrt{\log _{2}(4 / 3)} \approx 0.644$, then there is a constant $\gamma$ such that $f(n) \leq \gamma 2^{n-c \sqrt{n}}$.

Proof. Because $2^{c^{2}}<4 / 3$, we may choose $\delta \in\left(3 / 4,1 / 2^{c^{2}}\right)$. Let $\varepsilon=\delta-3 / 4$, let $k=\log _{2}\left(\varepsilon^{-2} \ln 4\right)$ as in Lemma 3.5 and let $n_{0}$ be large enough so that for all $n \geq n_{0}$ there is some $m$ as in Lemma 3.6 Note that because $\delta 2^{c^{2}}<1$, we may choose $\gamma$ to be large enough so that $(1+\gamma \delta) 2^{c^{2}} \leq \gamma$ holds and $f(n) \leq \gamma 2^{n-c \sqrt{n}}$ holds for all $n<n_{0}$. We prove that the bound holds for all $n$ by induction.

Let $n \geq n_{0}$, apply Lemma 3.6 to obtain $m$, and consider $T \in \mathcal{T}_{n}$ with root $r$. Let $T^{\prime}$ be the complete ternary subtree of $T$ rooted at $r$ with depth $m$. By induction, there exists a complete $S^{\prime} \in \mathcal{B}\left(T^{\prime}\right)$ with $\left|L\left(S^{\prime}\right)\right| \leq \gamma 2^{m-c \sqrt{m}}$.

For each $x \in\{0,1\}^{m}$, let $A_{x}$ be the set of leaves of $S^{\prime}$ with path-label $x$. We say that $x \in\{0,1\}^{m}$ is frequent if $\left|A_{x}\right| \geq 2^{c \sqrt{m}}$, and we say that $x$ is infrequent otherwise. Let $\alpha$ be the number of frequent labels, and let $\beta$ be the number of infrequent labels.

For each leaf $\sigma$ of $T^{\prime}$, let $T_{\sigma}$ be the complete ternary subtree of $T$ rooted at $\sigma$ of depth $n-m$. For each leaf $\sigma$ in $S^{\prime}$, we extend $S^{\prime}$ at $\sigma$ by selecting some $S_{\sigma} \in \mathcal{B}\left(T_{\sigma}\right)$. The choice for $S_{\sigma}$ depends on whether the path-label of $\sigma$ in $S^{\prime}$ is frequent or not. 
If $x$ is frequent, then for each $\sigma \in A_{x}$, we choose $S_{\sigma} \in \mathcal{B}\left(T_{\sigma}\right)$ arbitrarily. Otherwise, suppose that $x$ is infrequent, and let $\sigma_{1}, \ldots, \sigma_{r}$ be the leaves in $S^{\prime}$ with path-label $x$. Because $x$ is infrequent, we have $r \leq 2^{c \sqrt{m}}$. Moreover, each $T_{\sigma}$ has depth $n-m$ and $n-m \geq c \sqrt{m}+k \geq \log _{2}(r)+k$. Therefore Lemma 3.5 implies that there exist $S_{\sigma} \in \mathcal{B}\left(T_{\sigma}\right)$ such that $\left|\bigcup_{\sigma \in A_{x}} L\left(S_{\sigma}\right)\right| \leq \delta 2^{n-m}$. Gluing together all the trees $S_{\sigma}$ yields $S \in \mathcal{B}(T)$. We bound $|L(S)|$ as follows.

First, we bound the number of path-labels in $L(S)$ that extend frequent pathlabels. Note that by the definition of "frequent," $\alpha 2^{c \sqrt{m}} \leq 2^{m}$. If $x \in\{0,1\}^{m}$, then the total number of path-labels in $L(S)$ which extend $x$ is at most $2^{n-m}$. Hence the total number of path-labels in $L(S)$ which extend a frequent path-label is at most $\alpha 2^{n-m} \leq 2^{n-c \sqrt{m}}$.

Next, we bound the number of path-labels in $L(S)$ that extend infrequent pathlabels. If $x$ is not frequent, then the number of path-labels in $L(S)$ that extend $x$ is at most $\delta 2^{n-m}$. Note that $\beta \leq\left|L\left(S^{\prime}\right)\right| \leq \gamma 2^{m-c \sqrt{m}}$. Hence the number of path-labels in $L(S)$ that extend an infrequent path-label is at most $\beta \delta 2^{n-m} \leq$ $\gamma 2^{m-c \sqrt{m}} \delta 2^{n-m}=\gamma \delta 2^{n-c \sqrt{m}}$.

Adding these two bounds, we have that

$$
\begin{aligned}
|L(S)| & \leq(1+\gamma \delta) 2^{n-c \sqrt{m}} \\
& =(1+\gamma \delta) 2^{c(\sqrt{n}-\sqrt{m})} 2^{n-c \sqrt{n}} \\
& \leq(1+\gamma \delta) 2^{c^{2}} 2^{n-c \sqrt{n}} \\
& \leq \gamma 2^{n-c \sqrt{n}}
\end{aligned}
$$

as required.

\section{A LOWER Bound on $f(n)$}

Our strategy for bounding $f(n)$ from below is to construct edge-labeled ternary trees in which each path-label occurs along a limited number of paths, and then extend these trees slightly. 
12 englishROD DOWNEY, NOAM GREENBERG, CARL JOCKUSCH, AND KEVIN G. MILANS

Lemma 4.1. Define a sequence $\left\{a_{m}\right\}$ of integers via $a_{0}=1$ and $a_{m}=\left\lceil 3 a_{m-1} / 2\right\rceil$ for $m \geq 1$. For each $m$, there exists $T_{m} \in \mathcal{T}_{m}$ such that for each $x \in\{0,1\}^{m}$, the set $A_{x}$ of all leaves in $T_{m}$ with path-label $x$ satisfies $\left|A_{x}\right| \leq a_{m}$.

Proof. By induction on $m$. If $m=0$, the statement holds trivially. For $m \geq 1$, the inductive hypothesis implies that there is $T_{m-1} \in \mathcal{T}_{m-1}$ in which each path label occurs at most $a_{m-1}$ times. We extend $T_{m-1}$ to a complete ternary tree of depth $m$ as follows. Consider a path-label $x \in\{0,1\}^{m-1}$. At each vertex $u$ in $A_{x}$, add three children $v_{1}, v_{2}, v_{3}$ adjacent to $u$. Of the $3\left|A_{x}\right|$ new edges, arbitrarily label $\left\lceil 3\left|A_{x}\right| / 2\right\rceil$ with label 0 and label the others with label 1 . Repeating for each $x \in\{0,1\}^{m-1}$ yields $T_{m}$

It is straightforward to argue by induction that $(3 / 2)^{m} \leq a_{m} \leq 2(3 / 2)^{m}-1$. Solving the recurrence exactly has received some study. Odlyzko and Wilf showed that $a_{m}=\left\lfloor K(3 / 2)^{n}\right\rfloor$ where $K \approx 1.6222$ [18]; see also [4]. The sequence appears in the On-Line Encyclopedia of Integer Sequences with sequence identifier A061419. Our application requires only the easy upper bound $a_{m} \leq 2(3 / 2)^{m}$. By extending the trees provided in Lemma 4.1 we obtain a lower bound on $f(n)$.

Lemma 4.2. If $m \geq 0$ and $s=\left\lceil\log _{2} 2(3 / 2)^{m}\right\rceil$, then $f(m+s) \geq 2^{m}$.

Proof. Obtain $T_{m}$ as in Lemma 4.1 and let $s=\left\lceil\log _{2} 2(3 / 2)^{m}\right\rceil$. We obtain a tree $T \in \mathcal{T}_{m+s}$ by extending $T_{m}$ as follows. Fix some $x \in\{0,1\}^{m}$, and let $A_{x}$ be the set of all leaves in $T_{m}$ with path-label $x$. Because $\left|A_{x}\right| \leq 2(3 / 2)^{m}$, we may choose distinct labels $\theta(\sigma) \in\{0,1\}^{s}$ for each $\sigma \in A_{x}$. Extend $T_{m}$ at $\sigma$ by attaching the tree $T_{\sigma} \in \mathcal{T}_{s}$ with $L\left(T_{\sigma}\right)=\{\theta(\sigma)\}$. Following the same extension procedure for each label in $\{0,1\}^{m}$ yields $T$.

Consider $S \in \mathcal{B}(T)$ and let $\sigma_{1}, \ldots, \sigma_{r}$ be the vertices of $S$ at depth $m$. For each $\sigma_{j}$, let $\tau_{j}$ be a leaf in $S$ that is a descendant of $\sigma_{j}$. Because no two distinct leaves $\tau_{i}, \tau_{j}$ share a common path-label, we have that $|L(S)| \geq r=2^{m}$, as required.

Lemma 4.2 only yields a lower bound on $f(n)$ when $n$ is of a special form; however, we claim that for each $n$, either $n$ or $n-1$ is of a form to which Lemma 
4.2 applies. Let $b_{m}=m+\left\lceil\log _{2} 2(3 / 2)^{m}\right\rceil$, and note that for $m \geq 1$, we have that

$$
b_{m}-b_{m-1}=1+\left\lceil\log _{2} 2(3 / 2)^{m}\right\rceil-\left\lceil\log _{2} 2(3 / 2)^{m-1}\right\rceil<2+\log _{2} 3 / 2<3 .
$$

Because $b_{m}-b_{m-1}$ is an integer, we have that $b_{m}-b_{m-1} \leq 2$. We obtain the following general lower bound.

Theorem 4.3. For each $n$, we have $f(n) \geq 2^{\frac{n-3}{\log _{2} 3}} \geq(0.269) \cdot(1.548)^{n}$.

Proof. Let $m$ be an integer such that either $n$ or $n-1$ is equal to $m+\left\lceil\log _{2} 2(3 / 2)^{m}\right\rceil$.

Lemma 4.2 implies that $f(n) \geq f\left(m+\left\lceil\log _{2} 2(3 / 2)^{m}\right\rceil\right) \geq 2^{m}$. Note that

$$
n-1 \leq m+\left\lceil\log _{2} 2(3 / 2)^{m}\right\rceil \leq m+\left(\log _{2} 2(3 / 2)^{m}\right)+1=\left(\log _{2} 3\right) m+2
$$

and therefore $m \geq(n-3) / \log _{2} 3$.

Corollary 4.4. We have that $\lim _{n \rightarrow \infty}(f(n))^{1 / n} \geq 2^{\frac{1}{\log _{2} 3}} \geq 1.548$.

\section{An application to COMpUtability theory}

Our application requires a generalization to partial edge-labelings of the infinite ternary tree. A ternary sequence is a finite sequence of 0's, 1's, and 2's. The full ternary tree is the collection of all ternary sequences, ordered by sequence extension. This partial ordering can also be viewed as a (connected, acyclic) graph where two sequences are joined by an edge if one is an immediate extension of the other, that is, the one extends the other by one digit. The empty sequence is the root of the tree. The set of vertices at depth $k$ is $\{0,1,2\}^{k}$.

We consider partial edge-labelings of the full ternary tree. If $\sigma \tau$ is an edge in the full ternary tree and $\tau$ extends $\sigma$ by one character, then the level of $\sigma \tau$ is the depth of $\tau$. Hence, edges incident to the root are at level 1. Let $U$ be an infinite set of positive integers, which will indicate a set of levels of the full ternary tree; let $u_{1}, u_{2}, u_{3}, \ldots$ be an increasing enumeration of the elements of $U$. A $U$-edge-labeling of the full ternary tree is an assignment of a label in $\{0,1\}$ to each edge at every level in $U$. As before, reading the labels along edges in the path from the root to 
a vertex $\sigma$ gives a path-label, and reading the labels along the edges of an infinite path starting at the root gives a path-label in $\{0,1\}^{\omega}$, where $\omega=\{0,1,2, \ldots\}$. A binary subtree $S$ of the full ternary tree is complete if it has nonempty and has no leaves. (Note that we are considering subtrees in the graph-theoretic sense. In particular, such an $S$ is "2-bushy" in the sense that every node at depth $n$ has two children at depth $n+1$.) For such a subtree $S$, let $L(S)$ be the set of path-labels of paths through $S$, as before. Also as before, our object is to find such an $S$ with $L(S)$ "small." However, it is easily seen that it is not possible in general to choose such an $S$ with $L(S)$ countable. Instead, we ensure that $L(S)$ has measure 0 in the usual fair-coin measure on $2^{\omega}$. This amounts to choosing $S$ so that $\lim _{n}\left|L\left(S_{u_{n}}\right)\right| / 2^{n}=0$, where $S_{k}$ is the set of nodes and vertices of $S$ with depth at most $k$.

A set $U \subseteq \mathbb{N}$ is computable if there is an algorithm which, given $n \in \mathbb{N}$, decides if $n \in U$. A $U$-edge-labeling is computable if there is an algorithm which, given an edge $\sigma \tau$ in the full ternary tree, outputs the label on $\sigma \tau$. A full binary subtree $S$ of the full ternary tree is computable if there is an algorithm that, given a ternary sequence $\sigma$, decides if $\sigma$ is a vertex in $S$. For our application to computability theory, we also need the proof to be effective in the sense that we can choose $S$ to be computable if $T$ and $U$ are computable.

Theorem 5.1. Let $U$ be an infinite set of positive integers, and let $T$ be a $U$-edgelabeling of the full ternary tree. Then there is a complete binary subtree $S$ of $T$ such that $L(S)$ has measure 0 as a subset of $\{0,1\}^{\omega}$. Furthermore, if $U$ and $T$ are computable, we may require $S$ to be computable.

Proof. We prove the computable version of the result, and of course the other version follows by the same argument, omitting all mention of computability. Let $r$ be the root of $T$. We obtain $S$ by computing a sequence $S_{0}, S_{1}, \ldots$ of finite, complete binary subtrees rooted at $r$. Each tree $S_{j}$ is a proper subtree of $S_{j+1}$ and $L\left(S_{j}\right)$ has size at most $(3 / 4)^{j} \cdot 2^{n}$, where $n$ is the length of the path-labels in $S_{j}$. We set $S=\bigcup_{j} S_{j}$. Note that $S$ is a full binary subtree of $T$ and $L(S)$ has measure 0 . Moreover, $S$ is computable; to see if $\sigma$ is a vertex in $S$, simply compute $S_{j}$ for 
large enough $j$ so that path-labels in $S_{j}$ have length at least as long as $\sigma$ and test if $\sigma$ is in $S_{j}$.

Let $S_{0}$ be the binary subtree of depth 0 rooted at $r$. Given $S_{j}$, we show how to compute $S_{j+1}$. We obtain $S_{j+1}$ by gluing trees of the same depth to the leaves of $S_{j}$. These trees are obtained from a modified version of Lemma 3.5 . This modified version is explained next.

The argument of Lemma 3.5 easily extends to the partial edge-labeling case when the $n$ of the lemma is replaced by the length of the path-labels in the given partially edge-labeled trees. In fact, the argument becomes easier because we are no longer trying to establish a delicate upper bound on the number of labels. The key to applying the pigeon-hole principle in the proof of Lemma 3.5 is that $|\mathcal{X}|>2^{r}$, where $\mathcal{X}$ is a family of pairwise $\alpha$-orthogonal partitions of $\{0,1\}^{n}$. If we now set $\alpha=1$ and require $n>2^{r}$, we now achieve this easily by taking $\mathcal{X}=\left\{\left\{X_{i}, \overline{X_{i}}\right\}: 1 \leq i \leq n\right\}$, where $X_{i}$ is the set of binary words of length $n$ with a 1 in the $i$ th bit, so that $\mathcal{X}$ is a 1-orthogonal family of partitions of $\{0,1\}^{n}$. (This avoids the use of Chernoff's Inequality to construct a large $\alpha$-orthogonal family, at the cost of making $n$ much larger than in the original version of Lemma 3.5.) Since now $\alpha=1$, the first inequality in the final line of the proof of Lemma 3.5 yields $\left|\cup_{j} L\left(S_{j}\right)\right| \leq(3 / 4) 2^{n}$.

Let $m$ be the length of the path-labels in $S_{j}$, and let $A$ be the set of all leaves in $S_{j}$. Let $n=2^{|A|}+1$. For each $\sigma$ in $A$, let $T_{\sigma}$ be the complete ternary subtree rooted at $\sigma$ whose leaves have depth $u_{m+n}$ in $T$. Note that by construction, the path-labels in $T_{\sigma}$ all have length $n$.

Therefore, by the modified version of Lemma 3.5 discussed above, for each $\sigma \in A$, there exists a complete binary subtree $S_{\sigma}$ of $T_{\sigma}$ of full depth such that $\left|\bigcup_{\sigma \in A} L\left(S_{\sigma}\right)\right| \leq(3 / 4) 2^{n}$. Because $A$ is finite and there are only a finite number of candidates for each $S_{\sigma}$, we may compute such a collection of subtrees using brute force. Let $S_{j+1}$ be the binary subtree obtained by gluing $S_{\sigma}$ at each leaf $\sigma$ in $S_{j}$. Note that $S_{j+1}$ has depth $u_{m+n}$ and the path-labels in $S_{j+1}$ have length $m+n$. 
16 englishROD DOWNEY, NOAM GREENBERG, CARL JOCKUSCH, AND KEVIN G. MILANS

For each $x \in L\left(S_{j}\right)$, there are at most $(3 / 4) 2^{n}$ path-labels in $L\left(S_{j+1}\right)$ that extend $x$. It follows that $\left|L\left(S_{j+1}\right)\right| \leq(3 / 4) 2^{n} \cdot\left|L\left(S_{j}\right)\right|=(3 / 4) 2^{n}(3 / 4)^{j} 2^{m}=(3 / 4)^{j+1} 2^{m+n}$, as required.

Equip $\omega=\{0,1,2, \ldots\}$ with the discrete topology. The product space $\omega^{\omega}$, also known as Baire space, is a universal Polish space (a separable, completely metrizable space). Medvedev [11] considered subsets of Baire space to be "mass problems," where the idea is that the elements of a set $\mathcal{A}$ are the solution of the "problem." For example, if $\mathcal{A}$ is a singleton $\{f\}$, then the problem $\mathcal{A}$ is the problem of computing $f$. For another example, if $\mathcal{A}$ consists of all functions whose range is some nonempty set $X$, then $\mathcal{A}$ is the problem of enumerating the elements of $X$.

When is one mass problem $\mathcal{A}$ at least as difficult as another problem mass $\mathcal{B}$ ? Medvedev [11] introduced a reducibility on mass problems which is now often called Medvedev reducibility. Namely, $\mathcal{B}$ is Medvedev reducible to $\mathcal{A}$, denoted $\mathcal{B} \leq_{M} \mathcal{A}$, if there is a uniform way to compute a solution for $\mathcal{B}$ given any solution for $\mathcal{A}$. Formally, this means there is a Turing functional $\Phi$ such that $\Phi(f) \in \mathcal{B}$ for all $f \in \mathcal{A}$. In other words, there is a fixed oracle Turing machine which, given any function $f \in \mathcal{A}$ as oracle, computes a function $g \in \mathcal{B}$, which must of course be a total function. Note that Medvedev reducibility extends Turing reducibility in the sense that for $f, g \in \omega^{\omega}, g$ is Turing reducible to $f$ if and only if $\{g\}$ is Medvedev reducible to $\{f\}$.

The relation $\leq_{M}$ is a pre-partial ordering on Baire space. We call two mass problems Medvedev equivalent if each is Medvedev reducible to the other, and Medevedev equivalence is an equivalence relation. The equivalence classes are called Medvedev degrees; the collection of degrees is turned into a degree structure by adding the induced partial ordering. In fact, this degree structure is a distributive lattice, where 
the least upper bound is induced by pairwise effective join

$$
\mathcal{A} \times \mathcal{B}=\{f \oplus g: f \in \mathcal{A} \& g \in \mathcal{B}\} \quad 1
$$

and greatest lower bound given by effective disjoint union

$$
\mathcal{A} \sqcup \mathcal{B}=\{0 f: f \in \mathcal{A}\} \cup\{1 g: g \in \mathcal{B}\} .
$$

The Medvedev degrees have a least element $\mathbf{0}$ which consists of all mass problems that contain a computable function. The greatest element, the degree of the empty set, is usually ignored. The Medvedev degree of a mass problem $\mathcal{A}$ is denoted by $\operatorname{deg}_{M}(\mathcal{A})$ or sometimes simply a.

Medvedev reducibility is also known as strong reducibility. This is because Muchnik [15] later introduced a weaker version of Medvedev reducibility, the difference being that uniform computation is no longer required: a mass problem $\mathcal{B}$ is Muchnik (or weakly) reducible to a mass problem $\mathcal{A}$ if, for each $f \in \mathcal{A}$, there is a Turing functional $\Phi$ such that $\Phi(f) \in \mathcal{B}$. Here, the order of quantifiers allows for a different functional $\Phi$ for each $f \in \mathcal{A}$, and so the behavior of the reduction is no longer uniform over the functions in $\mathcal{A}$. The corresponding degree structure is isomorphic to the sublattice of the power set of the Turing degrees, consisting of all the sets of Turing degrees which are closed upwards, i.e. are unions of cones.

Now recall that Baire space $\omega^{\omega}$ is actually a topological space with basis $\left\{O_{\tau}: \tau \in\right.$ $\left.\omega^{<\omega}\right\}$, where $\omega^{<\omega}=\bigcup_{n \geq 0} \omega^{n}$ and the basic open set $O_{\tau}$ is given by $O_{\tau}=\{f \in$ $\omega^{\omega}$ : $f$ extends $\tau$ \}. Because $\tau$ is a finite list of numbers, algorithms can output the basic open set $O_{\tau}$ implicitly by referring to $\tau$.

The topological notions of open and closed sets can be refined using computability theory. We say that an open set $\mathcal{O} \subseteq \omega^{\omega}$ is effectively open (or $\Sigma_{1}^{0}$ ) if the collection of basic open subsets of $\mathcal{O}$ is computably enumerable. The complement of an effectively open set is effectively closed, or $\Pi_{1}^{0}$. We note that a set is effectively

\footnotetext{
${ }^{1}$ Where $(f \oplus g)(2 n)=f(n)$ and $(f \oplus g)(2 n+1)=g(n)$.
} 
closed if and only if it is the set of infinite paths through a computable subtree of $\omega^{<\omega}$.

Of particular interest are effectively closed sets which are computably bounded, in other words, are subsets of the subspace

$$
\prod_{n<\omega}\{0,1, \ldots, h(n)-1\}
$$

of $\omega^{\omega}$, for some computable function $h$. These closed sets are the sets of paths through trees which are finitely branching, and so are compact subsets of $\omega^{\omega}$; the extra effectiveness condition implies that in some sense these sets are "effectively compact." Computably bounded $\Pi_{1}^{0}$ classes have occurred as the set of solutions to problems in logic, combinatorics, and algebra and other areas; for a survey of this aspect of $\Pi_{1}^{0}$ classes, see [1, for example. It turns out that computably bounded $\Pi_{1}^{0}$ classes are Medvedev equivalent to effectively closed subsets of Cantor space $\{0,1\}^{\omega}=2^{\omega}$. Hence, much research was devoted to studying the Medvedev and Muchnik degrees of effectively closed subsets of Cantor space; see [19] for more details. Both substructures of the full degree structures are again distributive lattices, as the lattice operations, applied to effectively closed sets, yield effectively closed sets.

Let $\mathscr{P}_{M}$ be the lattice of Medevedev degrees of effectively closed subsets of Cantor space. In the lattice $\mathscr{P}_{M}$ there is a greatest element $\mathbf{1}$, which is the degree of the set of all consistent completions of Peano arithmetic, viewed as a mass problem via standard coding. Another $\Pi_{1}^{0}$ mass problem in 1 is the set $\operatorname{DNR}_{2}$ of $\{0,1\}$ valued diagonally nonrecursive functions. A function $f \in \omega^{\omega}$ is called diagonally nonrecursive if for all $e$ we have $f(e) \neq \varphi_{e}(e)$, where $\left\{\varphi_{e}\right\}_{e \in \omega}$ is an effective list of all partial computable functions. Here $f(e) \neq \varphi_{e}(e)$ means that either $\varphi_{e}(e)$ is undefined, or it is defined with a value unequal to $f(e)$.

The set DNR of all diagonally nonrecursive functions is effectively closed, but is not recursively bounded, indeed is not compact. However, we let, for every $k<\omega$, 
$\mathrm{DNR}_{k}$ be the set of all diagonally nonrecursive functions

$$
f: \omega \rightarrow\{0,1,2, \ldots, k-1\}
$$

i.e., $k$-valued diagonally nonrecursive functions. These sets are $\Pi_{1}^{0}$ and are recursively bounded, hence their Medvedev degrees lie in $\mathscr{P}_{M}$. As mentioned,

$$
\operatorname{deg}_{M}\left(\mathrm{DNR}_{2}\right)=\mathbf{1}
$$

is the greatest degree in $\mathscr{P}_{M}$. However, Jockusch [8, Theorem 6] showed that if we let $\mathbf{d}_{k}=\operatorname{deg}_{M}\left(\mathrm{DNR}_{k}\right)$, then

$$
\mathbf{d}_{2}>\mathbf{d}_{3}>\mathbf{d}_{4}>\ldots
$$

is a strictly decreasing sequence. This contrasts with Jockusch's result [8, Theorem 5]) that the Muchnik (weak) degrees of all of the classes $\mathrm{DNR}_{k}$ coincide, i.e., the classes $\mathrm{DNR}_{2}, \mathrm{DNR}_{3}, \ldots$ are all Muchnik equivalent.

If the classes $\mathrm{DNR}_{k}$ are "high", or close to 1 , then classes of positive measure should be considered "fat", and so "low", or close to $\mathbf{0}$. The measure we use is the product probability measure, using the fair coin measure on $\{0,1\}$. Simpson [19, Corollary 7.11] showed a non-join result: if $\mathbf{a} \in \mathscr{P}_{M}$ is the Medvedev degree of a $\Pi_{1}^{0}$ class of positive measure, $k \geq 2$, and $\mathbf{b} \in \mathscr{P}_{M}$ is not above $\mathbf{d}_{k}$, then the join $\mathbf{a} \vee \mathbf{b}$ is not above $\mathbf{d}_{k}$ either. Thus $\Pi_{1}^{0}$ classes of positive measure are so weak, that they cannot help any other $\Pi_{1}^{0}$ class compute bounded diagonally nonrecursive functions. Simpson asked [19, Remark 7.12] if the reason for this is that all $\Pi_{1}^{0}$ classes of positive measure are Medvedev reducible to each $\mathrm{DNR}_{k}$. Here we answer Simpson's question in the negative:

Theorem 5.2. There is a $\Pi_{1}^{0}$ class $P \subseteq 2^{\omega}$ of positive measure which is not Medvedev reducible to $\mathrm{DNR}_{3}$.

In fact, we prove a stronger result. Measure and $\Pi_{1}^{0}$ classes are closely tied to notions of effective randomness. For more background see [19]; here we just mention 
20 englishROD DOWNEY, NOAM GREENBERG, CARL JOCKUSCH, AND KEVIN G. MILANS

that every $\Pi_{1}^{0}$ class of positive measure contains a tail of every Martin-Löf random set $t^{2}$ (Kučera [10]), and that since the collection of Martin-Löf random sets is a $\Sigma_{2}^{0}$ set (an effective $F_{\sigma}$ set), there are nonempty $\Pi_{1}^{0}$ classes, necessarily of positive measure, which contain only Martin-Löf random sets. Thus in the Muchnik (weak) degrees, the degree of the set of Martin-Löf random sets is the same as the degree of any $\Pi_{1}^{0}$ class which contains only Martin-Löf random sets, is the greatest degree of $\Pi_{1}^{0}$ classes of positive measure. In the Medvedev degrees, the picture is not as tidy; the Medvedev degree $\mathbf{r}$ of the set of Martin-Löf random sets is not in $\mathscr{P}_{M}$, and in fact there is no greatest Medvedev degree of $\Pi_{1}^{0}$ classes of positive measure (Terwijn [17]). However, trivially, if $P$ is a $\Pi_{1}^{0}$ class which only contains Martin-Löf random sets, then the identity functional witnesses that $\mathbf{r} \leq \operatorname{deg}_{M}(P)$, and so Theorem 5.2 follows from the following theorem, which answers a question raised by J. Miller.

Theorem 5.3. $\mathbf{r} \not \leq \mathbf{d}_{3}$.

Indeed, we prove a little more. Recall that a set $X \in 2^{\omega}$ is Kurtz random (or weakly 1-random) if it is not a member of any null $\Pi_{1}^{0}$ subset of $2^{\omega}$. This is a notion of randomness which is much weaker than Martin-Löf randomness. We prove the following, which implies Theorem 5.3 .

Theorem 5.4. The set of Kurtz random sets is not Medvedev reducible to $\mathrm{DNR}_{3}$.

We note that in contrast, recently Greenberg and J. Miller [6] showed that the set of reals which have effective Hausdorff dimension 1 is Medvedev reducible to each $\mathrm{DNR}_{k}$.

Proof. Suppose for a contradiction that $\Phi$ is a Turing functional that witnesses that $\mathrm{DNR}_{3}$ is Medvedev reducible to the Kurtz random sets. Hence, for each $f \in \mathrm{DNR}_{3}$, we have that $\Phi(f)$ is the characteristic function of a Kurtz random set and is thus

${ }^{2}$ Recall that a sequence $X \in 2^{\omega}$ is Martin-Löf random if whenever $\left\langle U_{n}\right\rangle$ is an effective sequence of effectively open subsets of $2^{\omega}$ such that the measure of each $U_{n}$ is at most $2^{-n}$, we have $X \notin \bigcap_{n} U_{n}$. Equivalently, the initial segments of $X$ are incompressible, in the sense that there is a constant $c$ such that for all $n, K(X \uparrow n) \geq n-c$; here $K$ denotes prefix-free Kolmogorov complexity. See, for example, [2] for more details on effective randomness. 
total (i.e. given an input, returns a value after a finite amount of time). Without loss of generality, we may assume that for all $f \in 3^{\omega}, \Phi(f)$ is total and $\{0,1\}$ valued. We do this by replacing $\Phi$ by a modified Turing functional which, with oracle $f \in 3^{\omega}$ and input $n$, simulates $\Phi$ until either $\Phi(f)(n)$ converges or it is discovered by a systematic search that $f(e)=\varphi_{e}(e)$ for some $e$, so $f \notin \mathrm{DNR}_{3}$. One of these events must occur since $\Phi(f)$ is total for all $f \in \mathrm{DNR}_{3}$. If the former occurs first, the modified functional outputs $\Phi(f)(n)$, and otherwise it outputs 0 (say).

By compactness, and buffering the use of $\Phi$-computations, we can obtain an effective increasing sequence $u_{1}<u_{2}<u_{3}<\ldots$ such that for all $n \geq 1$, for all $X \in 3^{\omega}$, the $X$-use of computing $\Phi(X)\left\lceil n\right.$ is exactly $u_{n}$. Let $U=\left\{u_{1}, u_{2}, u_{3}, \ldots\right\}$. Thus $\Phi$ yields a computable $U$-edge labeling of the full ternary tree $3^{<\omega}$ : for $\sigma \in$ $\{0,1,2\}^{u_{n}}$, we let $\Phi(\sigma) \in\{0,1\}^{n}$ be the result of applying $\Phi$ to the oracle $\sigma$, and so we label the parent edge incident to $\sigma$ in the full ternary tree with the last bit of $\Phi(\sigma)$. That is, the path-label of $\sigma$ is exactly $\Phi(\sigma)$ for all $\sigma \in \bigcup_{n \geq 1}\{0,1,2\}^{u_{n}}$, and hence for all $f \in 3^{\omega}, \Phi(f)$ is the path-label of $f$.

By Theorem 5.1, there is a computable full binary subtree $S$ of $3^{<\omega}$ such that $L(S)$ is null in Cantor space. We show that $L(S)$ is effectively closed by enumerating the basic open subsets of its complement $\{0,1\}^{\omega}-L(S)$. For each $n \geq 1$, compute $u_{n}$ and compute the set $A$ of all vertices in $S$ at depth $u_{n}$. Next, for each $\sigma \in A$, compute the path-label of $\sigma$. In the enumeration, output all basic open sets $O_{\tau}$ such that $\tau \in\{0,1\}^{n}$ is not the path-label of some $\sigma \in A$. It follows that $L(S)$ is a null $\Pi_{1}^{0}$ class.

Let $T$ be the subtree of $3^{<\omega}$ induced by the vertices $\tau$ such that $\mathrm{DNR}_{3}$ contains an extension of $\tau$. Note that if $\tau$ is in $T$, then because $\tau$ has three children in $3^{<\omega}$ and at most one violates the diagonally non-recursive condition, at least two its children are in $T$. It follows that $T$ and $S$ contain a common infinite path $f \in\{0,1,2\}^{\omega}$. Because $f$ is an infinite path in $T$, we have that $f$ is in $\mathrm{DNR}_{3}$. Because $f$ is an infinite path in $S$, we have that $\Phi(f) \in L(S)$, and so $\Phi(f)$ is a member of a null $\Pi_{1}^{0}$ class, which implies that $\Phi(f)$ is not Kurtz random. Hence 
22 englishROD DOWNEY, NOAM GREENBERG, CARL JOCKUSCH, AND KEVIN G. MILANS

$f \in \mathrm{DNR}_{3}$ but $\Phi(f)$ is not Kurtz random, and so $\Phi$ fails to witness that the class of Kurtz random sets is Medvedev-reducible to $\mathrm{DNR}_{3}$, as required.

\section{REFERENCES}

[1] D. Cenzer and J.B. Remmel, $\Pi_{1}^{0}$ classes in mathematics, pp. 623-681 in Handbook of Recursive Mathematics, edited by Y.L. Ershov, S.S. Goncharov, A. Nerode, and J.B. Remmel, Studies in Logic and the Foundations of Mathematics, North Holland, 1998.

[2] R. Downey and D. Hirschfeldt, Algorithmic Randomness and Complexity, SpringerVerlag, in preparation.

[3] R. Downey, D.R. Hirschfeldt, A. Nies, and S. Terwijn, Calibrating randomness, Bull. Symbolic Logic 12 (2006), 411-491.

[4] S.R. Finch, Powers of 3/2 modulo one, Mathematical Constants (2003), 194-199.

[5] R. Goldblatt, The McKinsey axiom is not canonical, J. Symbolic Logic 56 (1991), $554-562$.

[6] Noam Greenberg and J.S. Miller, Diagonally non-recursive functions and effective Hausdorff dimension, in preparation.

[7] Zoltán Füredi, Carl G. Jockusch, Jr., and Lee A. Rubel, Difference sets and inverting the difference operator, Combinatorica, 16(1) (1996), 87-106, .

[8] C.G. Jockusch, Degrees of functions with no fixed points, in Logic, Philosophy, and Methodology of Science VIII, edited by J. E. Fenstad, I. T. Frolov, and R. Hilpinen, North-Holland, Amsterdam, New York, Oxford, Tokyo, 1989, 191-201.

[9] Henry A. Kierstead, An effective version of Dilworth's theorem, Trans. Amer. Math. Soc., 268(1) (1981), 63-77.

[10] A. Kučera. Measure, $\Pi_{1}^{0}$-classes and complete extensions of PA, in Recursion Theory Week (Oberwolfach, 1984), volume 1141 of Lecture Notes in Math., pages 245-259, Springer, Berlin, 1985.

[11] Yu. T. Medvedev, Degrees of difficulty of the mass problem, Dokl. Akad. Nauk SSSR (N.S.), 104 (1955), 501-504. .

[12] Joseph R. Mileti, The canonical Ramsey theorem and computability theory, Trans. Amer. Math. Soc., 360(3) 2008, 1309-1340. 
[13] Antonio Montalbán. On the equimorphism types of linear orderings, Bull. Symbolic Logic, 13(1) (2007), 71-99.

[14] R. Motwani and P. Raghavan, Randomized Algorithms, Cambridge University Press, Cambridge, New York, 1995.

[15] A. A. Mučnik. On strong and weak reducibility of algorithmic problems, Sibirsk. Mat. Ž., 4 (1963), 1328-1341.

[16] G. Pólya and G. Szegö, Problems and Theorems in Analysis I, Springer-Verlag, Berlin, Heidelberg, New York, 1987.

[17] Sebastiaan A. Terwijn, The Medvedev lattice of computably closed sets, Arch. Math. Logic, 45(2) (2006), 179-190.

[18] A.M. Odlyzko and H.S. Wilf, Functional iteration and the Josephus Problem, Glasgow Math. J. 33 (1991), 235-240.

[19] S.G. Simpson, Mass problems and randomness, Bull. Symbolic Logic 11 (2005), 1-27.

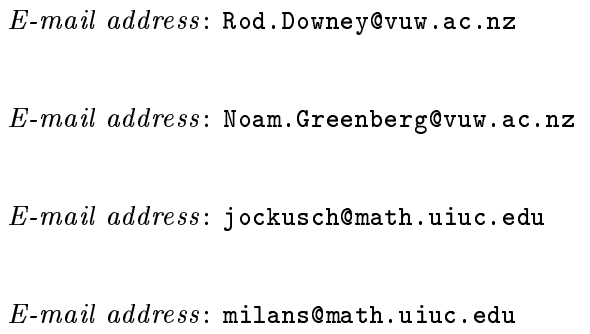

